\title{
Intra-species Transduction with Proteus mirabilis High Frequency Transducing Phages
}

\author{
By J. N. COETZEE \\ Department of Microbiology, University of Pretoria, South Africa
}

(Received 8 August 1975)

\begin{abstract}
SUMMAR Y
The properties of three additional Proteus mirabilis hosts for the high frequency transducing (HFT) phages 5006MHFTk and 5006MHFTak are described. The phages transduce resistance to kanamycin and to ampicillin plus kanamycin, respectively, and were produced by ultraviolet induction of derivatives of $P$. mirabilis strain PM5006. Strain PM804 could not be shown to adsorb the phages although it yielded a few transductants. All transductants, even those produced at low multiplicities of phage input, were lysogenic and segregated markers at high frequency. Ultraviolet induced phage lysates of these transductants transduced PM804 at higher frequencies and PM5006 at lower frequencies than the original phages. Strain PM804 or its derivatives did not adsorb phage from these lysates. Transmission experiments through PM5006 of phage in the transductant lysates confirmed that PM804 had a host-controlled modification system which modified HFT phage from PM5006. That PM804 also possessed a restriction system was inferred from the greater numbers of transductants obtained with phage which bore a compatible modification pattern. Strain $\mathrm{N}$ and a restrictionless mutant of it named NHI, adsorbed the HFT phages avidly and failed to modify their host range. Transduction frequencies of the phage markers were about $10^{-4} /$ p.f.u. adsorbed to strain NHI and only about tenfold lower to strain N which did not plate the phages. Transductants also had the features of heterogenotes and those obtained at low multiplicities of infection were non-lysogenic. The latter transductants adsorbed homologous phage while NHI transductants also plated the HFT lysates. Strain NHI, lysogenized by the parent phage $5006 \mathrm{M}$, did not adsorb the HFT phages. These findings suggest that the HFT phages were most likely defective for lysogenic conversion to homologous phage non-adsorption. The postulated restriction enzymes of PM804 and strain N could not be shown to be thermolabile.
\end{abstract}

\section{INTRODUCTION}

High frequency transducing (HFT) phages for Proteus mirabilis strain PM5006 have been described (Coetzee, 1974, 1975a). The first was derived by u.v. irradiation of a kanamycinresistant transductant of PM5006 produced by phage 34 (Coetzee \& Sacks, 1960) transduction of plasmid R394 (Coetzee, Datta \& Hedges, 1972). It is now named phage 5006MHFTk (Coetzee, $1975 b$ ). The other lysate was produced by irradiation of a particular ampicillinand kanamycin-resistant clone of PM5006 produced by conjugal transfer of $\mathrm{R}_{394}$ to the abovementioned kanamycin-resistant transductant. This phage is named phage 5006MHFTak (Coetzee, 1975b).

Proteus mirabilis strains PM5006 and PMI 3 harbour a cryptic prophage 5006M (Krizsanovich, 1973) which is similar to phage 34 and serologically identical to both the HFT phages. The presence of this prophage complicated analysis of PM5006 transductants by the HFT phages (Coetzee, 1974, 1975a) and thus a prophage-free susceptible host was required. 
High frequency transduction of antibiotic resistance provides a means of selection for transductants from bacterial strains not normally sensitive to the phage (Goldberg, Bender $\&$ Streicher, 1974). Coetzee (1975b) reported that a $P$. vulgaris strain PVI 27 could be transduced to kanamycin resistance by the $P$. mirabilis HFT phages. Strain PVI27 was not an entirely satisfactory host as it adsorbed the phages poorly, did not plate them and was ampicillin-resistant. Nevertheless, heterogenote-like transductants (Morse, Lederberg \& Lederberg, 1956; Luria, Adams \& Ting, 1960) formed at low multiplicities of phage input, adsorbed homologous phage. This was a unique situation because strains PMI 3 and PM5006 lysogenized by phage $5006 \mathrm{M}$ did not adsorb this phage. The latter phenomenon was due to lysogenic conversion to homologous phage non-adsorption (Coetzee, 196I), possibly produced by the interaction of resident cryptic and superinfecting prophage (see Krizsanovich, I973). This raised the possibility that the HFT phages were defective in the converting function but, as PVI27 did not plate phage $5006 \mathrm{M}$, an essential control was missing.

Because the ampicillin resistance marker of phage 5006MHFTak would provide the necessary selection for retransduction of phage 5006MHFT $k$ transductants if the latter adsorbed the phage, a large number of ampicillin- and kanamycin-sensitive strains of $P$. vulgaris and $P$. mirabilis were screened by the method previously used (Coetzee, $1975 b$ ) as possible hosts for the phages (J. N. Coetzee, unpublished). One P. mirabilis strain, PM804, yielded transductants. Also available were $P$. mirabilis strain $\mathrm{N}$, which appeared to restrict phage 34.PMI 3 without modifying it, and a restrictionless mutant of $\mathrm{N}$, named NHI (Coetzee \& Smit, 1969). Phage 5006M.PM5006 behaves like phage 34.PMI 3 in strain N (unpublished observations) and this paper describes results of transductions of antibiotic resistance markers to $P$. mirabilis strains PM804, N and NHI by phages 5006MHFT $k$ and 5006MHFTak.

\section{METHODS}

Bacteria and phages. These are presented in Table I.

Media. The sources of the nutrient broth, nutrient agar and MacConkey agar have been described (Coetzee et al. 1973). Minimal medium was that of Grabow \& Smit (I967). Selection for drug resistance was made on MacConkey agar containing ampicillin and/or kanamycin at $50 \mu \mathrm{g} / \mathrm{ml}$ or streptomycin at $\mathrm{I} \mathrm{mg} / \mathrm{ml}$. Incubation temperature was $37^{\circ} \mathrm{C}$.

General phage techniques. These were according to Adams (1956) and Coetzee (I974). Freeze-dried phage 34 antiserum (Coetzee \& Sacks, 1960) with a $K_{m}$ value of I $80 \mathrm{~min}^{-1}$ against phages 5006MHFT $k$ and 5006MHFTak was used. Particular care was taken to ensure that phage lysates were sterile: after shaking thoroughly with $\mathrm{O} \cdot \mathrm{I}$ vol. chloroform the phage was decanted into sterile bottles; after overnight incubation, $10 \mathrm{ml}$ samples were removed into $100 \mathrm{ml}$ broth and incubated for $48 \mathrm{~h}$ to test for sterility.

Preparation of bacteria. Unless mentioned otherwise, bacteria in the stationary phase of growth were used. Overnight broth cultures were resuspended in $0.0 \mathrm{I} \mathrm{M}$-tris buffer $\mathrm{pH} 7 \cdot 3$ with $0.0 \mathrm{I} \mathrm{M}-\mathrm{MgSO}_{4}$, and starved for $30 \mathrm{~min}$. Exponentially-growing cells were obtained by diluting an overnight broth culture $\mathrm{I}: 5$ in pre-warmed broth and incubating for $40 \mathrm{~min}$.

Concentration of phage lysates. The method described for $\mathrm{P} 22$ by Yamamoto \& Alberts (1970) was used.

Phage adsorption. The method of Kondo \& Mitsuhashi (I966) was used. Phage (0. I ml) was added to $2 \mathrm{ml}$ of the streptomycin-sensitive test strain prepared as above at different multiplicities of input (m.i.) of plaque-forming units (p.f.u.). At time zero and at intervals samples were removed, diluted in saline and titrated on PM5006 str-r using media which contained I $\mathrm{mg}$ streptomycin $/ \mathrm{ml}$. The viable count of the test strain was done.concurrently 
Table I. Bacteria and phages

All strains were sensitive to ampicillin and kanamycin.

Bacteria

Proteus mirabilis PM804

P. mirabilis PM5006

P. mirabilis PM5006 str-r

P. mirabilis $\mathrm{N}$

$P$. mirabilis $\mathrm{N}$ met-I

$P$. mirabilis NHI

P. mirabilis $\mathrm{NHI}(5006 \mathrm{M})$

Bacteriophages

5006M.PM5006 str-r

5006MHFT $k$

5006MHFTak
Properties

Isolated locally from human faeces. Swarms form lines of demarcation with those of PM5006, strain $\mathrm{N}$ and $\mathrm{NHI}$

Host for phages used. Cryptically lysogenic for phage $5006 \mathrm{M}$. Not susceptible to bacteriocin or phage 13 vir liberated by strain $\mathrm{N}$

Spontaneous mutant resistant to I $\mathrm{mg}$ streptomycin/ml

Adsorbs phages 34.PMI 3 and 5006M.PM5006 well but restricts without modifying the phage. Lysogenic for phage $13 \mathrm{vir}$ and produces a phage tail-like bacteriocin. Forms Dienes demarcation line with PM5006

Auxotrophic mutant of strain N

Mutant of strain N which plates phage 34.PMI 3 and phage 5006M.PM5006 with an efficiency of $5 \times 10^{-1}$

Strain NHI lysogenized with phage 5006M. Converted to homologous phage nonadsorption

Cryptic prophage of PM5006. Lysate produced by lytic infection of PM5006 str-r. On lysogenization converts hosts PMI 3 and PM5006 to non-adsorption of homologous phage

High frequency transducing for kanamycin resistance. Produced by u.v. induction of PM5006( $(\overline{\mathrm{R} 394})$ * Serologically identical to phage $5006 \mathrm{M}$

High frequency transducing phage for ampicillin and kanamycin resistance. Produced by u.v. induction of PM5006 $\overline{\overline{\left(\mathrm{R}_{394}\right.}} \boldsymbol{} \uparrow$.

Serologically identical to phage $5006 \mathrm{M}$
Reference

Coetzee (unpublished)

Dienes (1946, 1947)

Coetzee \& Smit ( 1969 , I970)

Coetzee (1974, 1975a)

Krizsanovich (1973)

Coetzee (1974)

Coetzee \& Sacks ( I 960)

Coetzee \& Smit ( 1969 )

Coetzee, De Klerk \&

Coetzee (1968)

Coetzee \& Smit (1969)

Coetzee \& Smit (I 969)

Coetzee (unpublished)

Coetzee (196I)

Krizsanovich (1973)

Coetzee (1974)

Coetzee \& Sacks (1960)

Coetzee (1974, 1975b)

Coetzee et al. (1973)

Coetzee (I975 $a, b)$

* Superior line indicates that markers of R factor R394 were transduced to PM5006.

$\dagger$ Superior lines indicate that markers of R factor R394 were transduced to a derivative of PM5006(R $\overline{394})$.

on MacConkey agar. Various concentrations of the test organisms in different phases of growth were used, and some tests were done at $15,25,30$ and $37{ }^{\circ} \mathrm{C}$.

Selection of auxotrophic mutants of PM804 and NHI. The $N$-methyl- $N^{\prime}$-nitro- $N$-nitrosoguanidine method of Adelberg, Mandel \& Chen (1965) was used.

Ultraviolet irradiation of phage lysates. The method of Coetzee (1974) was used.

Phage induction. Ultraviolet light induction was according to Coetzee (1974) and cultures were treated with mitomycin C according to Altenbern \& Stull (I965).

Transduction. The Millipore membrane filter technique (Coetzee, I974) was used. The phage suspension was added to the bacterial culture and the mixture incubated for $15 \mathrm{~min}$ before dilution in saline and filtration. Membranes with impinged bacteria were placed directly on minimal medium (transduction to prototrophy), on pre-warmed nutrient agar for $\mathrm{I} \mathrm{h}$ (transduction of ampicillin or kanamycin resistance) or $3 \mathrm{~h}$ (transduction of 
streptomycin resistance) before transfer to selective media. In experiments where prevention of lysogenization of transductants by phage liberated during the experiment was critical, phage antiserum was added to the adsorption mixture 10 min after addition of phage. After a further $10 \mathrm{~min}$ the adsorption mixture was filtered with a large excess of saline. In experiments to exclude transfection, the adsorption mixture contained $6 \mu \mathrm{g}$ deoxyribonuclease I (Sigma) $/ \mathrm{ml}$. Transductant clones were streaked for single colonies on selective media before further investigation. Some transductant clones were also suspended in phage antiserum for $\mathrm{l} \mathrm{h}$ before streaking for single colonies.

Single cycle growth of phage. This was done according to the method of Coetzee \& Smit (1969). Phage was adsorbed to about $2 \times 10^{9}$ organisms for 10 min at $37{ }^{\circ} \mathrm{C}$. A sample was then diluted $\mathrm{I}: 100$ in chloroform broth and titrated on PM5006 for unadsorbed phage. Another sample was diluted $\mathrm{I}: 2$ in phage antiserum. After $5 \mathrm{~min}$ at $37^{\circ} \mathrm{C}$ two samples were removed from the serum tube. One was diluted into chloroform broth for free phage assay while the other was diluted into nutrient broth and titrated for infective centres on a particular host.

Heat treatment of bacteria. This was done according to the methods of Cornelis \& Colson ( I975) and Lederberg (1965). A number of $3 \mathrm{ml}$ samples of an overnight broth culture in thin-walled glass tubes were centrifuged and the bacteria from each sample resuspended in I $\mathrm{ml}$ of $0.85 \%$ saline pre-heated to the chosen temperature. The tubes were maintained in a water bath at this particular temperature. At intervals, duplicate tubes were brought to $37^{\circ} \mathrm{C}$ in a water bath for $2 \mathrm{~min}$; the bacteria in one of the tubes were used immediately as recipients in transduction experiments, while single-cycle phage growth experiments were conducted with the cultures in the other tube.

\section{RESULTS \\ Adsorption and plating efficiencies of phages}

Strain PM804 and its derivatives did not adsorb phage as measured by any of the methods used (Table 2) and viable counts of test and control cultures were similar (not shown). Phage grown on PM804 transductants plated on PM5006 and NHI but adsorption to PM804 could not be demonstrated. Strains N and NHI adsorbed the phages to the same extent as PM5006. High titre phage formed areas of clearing when spotted on strain $\mathrm{N}$ but plaques were not observed. This was investigated in a single-cycle phage growth experiment (Table 3 , line 1). Phage from strain $\mathrm{N}$ did not plate on it and the organism restricted the phage without modifying it. Strain NHI plated the phages with an efficiency (e.o.p.) of about $5 \times 10^{-1}$ (Table 2) and lysogenic derivatives were readily obtained. This small reduction in e.o.p. was unlikely to result from restriction (see Coetzee \& Smit, 1969). Phages grown on strain NHI plated with an e.o.p. of one on PM5006 but only formed areas of clearing and no plaques when spotted on strain N. Phage derived from NHI transductants could not be shown to adsorb to NHI lysogenic transductants or to NHI lysogenized by phage $5006 \mathrm{M}$, but adsorbed avidly to NHI non-lysogenic transductants and plated with an efficiency of one on PM5006 (Table 2). Strain NHI did not restrict or modify the HFT phages from PM5006 (see Table 3, line 2).

\section{Transduction}

A few transductants were obtained using PM804 as a recipient for the HFT phages (Table 4 , lines I to 6). The phage lysates were sterile and uninfected recipient controls did not grow on selective media. Properties of the clones also ruled out a mutational origin and in experiments with phage 5006MHFTak lysates, it was unlikely that mutants resistant to two 
Table 2. Phage adsorption and plating efficiencies on P. mirabilis strains

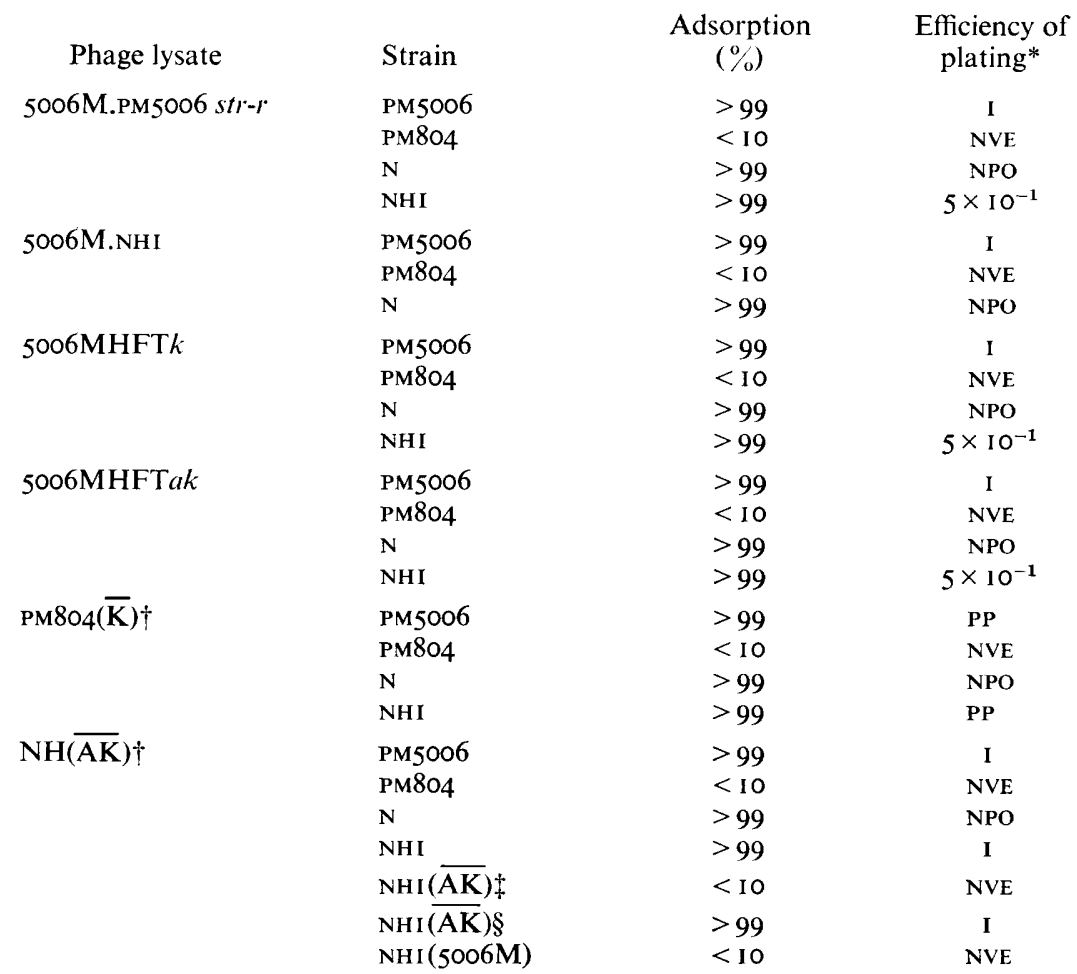

NPO, When spotted on lawns of $\mathrm{N}$, the phage caused areas of partial clearing which disappeared with higher dilutions of the phage; no plaques observed; NVE, no visible effect; PP, plaques present but efficiency of plating not determined as phage did not plate on PM804; K, resistance to kanamycin; AK, resistance to ampicillin and kanamycin.

* Plaque-forming titre on strain/titre on last host.

$\uparrow$ Superior line indicates that markers were transduced.

$¥$ Lysogenic transductant.

$\S$ Non-lysogenic transductant.

\section{Table 3. Single cycle growth of a phage 5006 MHFTak lysate in strains $\mathrm{N}$ and $\mathrm{NHI}$}

Phage was adsorbed to $4 \times 10^{9}$ organisms of strain $\mathrm{N}$ and $9 \times 10^{8}$ strain NHI bacteria for $10 \mathrm{~min}$ at $37^{\circ} \mathrm{C}$. A sample was then diluted $\mathrm{I}: \mathrm{I} 00$ in broth containing $0 . \mathrm{I}$ vol. chloroform and titrated on strain PM5006 for unadsorbed phage. Another sample was diluted $1: 2$ in phage antiserum. After $5 \mathrm{~min}$ at $37^{\circ} \mathrm{C}$ two samples were removed from the serum tube. One sample was diluted into chloroform broth for free phage assay. The other was diluted $\mathrm{I}: \mathrm{I} 00$ in broth and titrated for infective centres on strains PM5O06 and N or NHI.

\begin{tabular}{|c|c|c|c|c|c|c|}
\hline \multirow[b]{2}{*}{ Strain } & \multirow[b]{2}{*}{ M.o.i. } & \multirow{2}{*}{$\begin{array}{l}\text { Adsorbed } \\
\text { phage }(\%)\end{array}$} & \multirow{2}{*}{$\begin{array}{c}\text { Free phage } \\
\text { after antiserum }\end{array}$} & \multicolumn{2}{|c|}{$\begin{array}{l}\text { No. infective } \\
\text { centres on: }\end{array}$} & \multirow{2}{*}{$\begin{array}{l}\text { infected } \\
\text { bacteria } \\
\text { producing } \\
\text { centres on } \\
\text { PM5006 }\end{array}$} \\
\hline & & & & PM5006 & $\mathrm{N}$ & \\
\hline $\mathbf{N}$ & 0.4 & $99 \cdot 6$ & $<10^{2}$ & $\mathrm{I} \cdot 3 \times \mathrm{IO}^{4}$ & $<\mathrm{IO}^{2}$ & $10^{-5}$ \\
\hline NHI & 0.5 & $99 \cdot 6$ & $<10^{2}$ & $2.7 \times 10^{8}$ & $<10^{2}$ & I \\
\hline
\end{tabular}


Table 4. Transduction of antibiotic resistance by HFT phages to strains of P. mirabilis

One $\mathrm{ml}$ of a stationary phase suspension of bacteria was mixed with $0 . \mathrm{I} \mathrm{ml}$ phage lysate and incubated for 15 min before dilution in saline and filtration through Millipore membranes. Membranes with impinged bacteria were placed directly on minimal medium for transductions to prototrophy or on nutrient agar for $\mathrm{I} h$ for transductions of ampicillin and kanamycin resistance and for $3 \mathrm{~h}$ on nutrient agar for streptomycin resistance transductions, before transfer to selective media.

\begin{tabular}{|c|c|c|c|c|c|c|}
\hline Transducing lysate & Recipient & M.o.i. & $\mathbf{K}$ & AK & $s t r-r$ & Prototrophy \\
\hline $5006 \mathrm{MHFT} k$ & PM804 & $0.9^{*}$ & 2 & - & - & - \\
\hline $5006 \mathrm{MHFT} k$ & PM804 & $7 \cdot I^{*}$ & 3 & - & - & - \\
\hline $5006 \mathrm{MHFT} k$ & $\mathrm{PM} 804$ met-I & $7 \cdot 3^{*}$ & 2 & - & - & 0 \\
\hline 5006MHFTak & PM804 & $0.8^{*}$ & I & 0 & - & - \\
\hline $5006 \mathrm{MHFT} a k$ & PM804 & $8 \cdot 1^{*}$ & 2 & I & - & $-\cdots$ \\
\hline $5006 \mathrm{MHFT} a k$ & PM804 arg-I & $8 \cdot 0^{*}$ & I & 3 & 一 & 0 \\
\hline $5006 \mathrm{MHFT} k$ & $\mathbf{N}$ & 0.8 & $10^{-5}$ & - & - & - \\
\hline 5006MHFTak & $\mathrm{N}$ & $I \cdot I$ & - & $4 \times 10^{-\overline{5}}$ & - & - \\
\hline $5006 \mathrm{MHFT} k$ & M met-I & $3 \cdot 1$ & $9 \times 10^{-4}$ & - & - & $10^{-8}$ \\
\hline $5006 \mathrm{MHFT} k$ & & 0.9 & & & & \\
\hline+ & $\mathrm{N}$ & & $3 \times 10^{-5}$ & - & - & - \\
\hline 5006М.PM5006 str-r & & 3.0 & & & & \\
\hline $5006 \mathrm{MHFT} k$ & NHI & 0.8 & $10^{-4}$ & 一 & - & 一 \\
\hline 5006MHFTak & NHI $m e t-I$ & $2 \cdot 8$ & $2 \times 10^{-4}$ & $2 \times 10^{-4}$ & - & $2 \times 10^{-7}$ \\
\hline 5006MHFTak & & 0.7 & & & & \\
\hline+ & NHI & & - & $6 \times 10^{-4}$ & - & - \\
\hline 5006M.PM5006 str-r & & $3 \cdot 0$ & & & & \\
\hline 5006М.PM5006 str-r & NHI & $3 \cdot 0$ & - & - & $8 \times 10^{-7}$ & 一 \\
\hline 5006M.PM5006 str-r & PM5006 & $3 \cdot 2$ & - & - & $8 \times 10^{-7}$ & - \\
\hline
\end{tabular}

M.o.i., multiplicity of infection; other symbols as in Table 2.

* Multiplicity input.

antibiotics would be selected. Transfection was excluded, by finding that the appearance of the transductants was not influenced by the presence of DNAase. Many experiments did not yield PM804 transductants but this erratic behaviour could often be overcome by increasing the m.i. to about 8 . This procedure did not necessarily increase the number of transductants obtained. Experiments with recipient cells in the exponential phase of growth did not affect the predictability or the number of transductants obtained. Abortive transductants were not detected and phage 5006M.PM5006 str-r at various m.i. had no effect on the transductions. This was probably due to poor adsorption which prevented simultaneous infection. The HFT phage lysates are capable of general transduction at low frequencies (Coetzee, 1974, 1975a) but, as expected, could not transduce auxotrophs of PM804 to prototrophy (Table 4 , lines 3 and 6).

Strain $\mathrm{N}$ was transduced to antibiotic resistance at frequencies of $\mathrm{I} \times \mathrm{IO}^{-5}$ to $4 \times \mathrm{IO}^{-5} /$ p.f.u. adsorbed (Table 4 , lines 7 and 8 ) in comparison to frequencies of about $5 \times 10^{-2} /$ p.f.u. adsorbed for transductions to PM5006 (Coetzee, I974, I975a). With strain NHI as recipient, transduction frequencies were raised about tenfold to $\mathrm{I} \times 10^{-4}$ to $2 \times 10^{-4} /$ p.f.u. adsorbed (Table 4, lines I I and 12) and transductants were obtained at multiplicities of infection (m.o.i.) as low as $5 \times 10^{-5}$. The general transducing phage $5006 \mathrm{M}$ transduced both strain $\mathrm{N}$ and PM5006 to streptomycin resistance at frequencies of $8 \times 10^{-7} /$ p.f.u. adsorbed (Table 4 , lines 14 and 15 ). Why the HFT lysates did not transduce NHI to antibiotic resistance at frequencies similar to those at which they transduced PM5006 is not known. There was a small difference in the e.o.p. of the phages on PM5006 and NHI (Table 2) but this could 


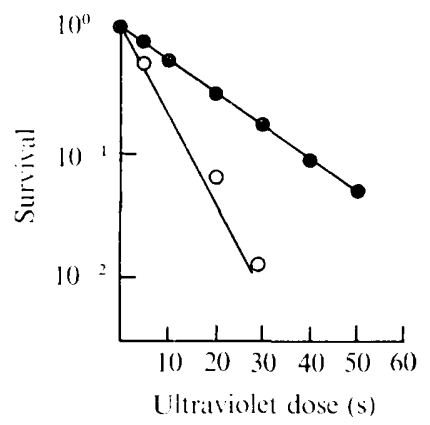

Fig. I. Effect of u.v. irradiation on a phage 5006MHFTak lysate. Quantitative transduction experiments were done with the irradiated lysates at a m.o.i. of $1 \cdot 2$ p.f.u. of the unirradiated lysate. Strain $\mathrm{NHl}$ was the recipient. Transducing activity; $\mathrm{O}$, plaque formation.

hardly explain the differences encountered, and abortive transductants were not found. Simultaneous injection with phage 5006M.PM5006 str-r increased transduction frequencies for HFT phages in strains $\mathrm{N}$ and NHI about threefold (Table 4, lines IO and I3). Maximal effects with the former phage were reached at a m.o.i. of about 3. Auxotrophic mutants of strain NHI were readily obtained and these, together with a mutant of strain $\mathrm{N}$, were transduced to prototrophy by the HFT phage lysates at low frequencies, while transduction of

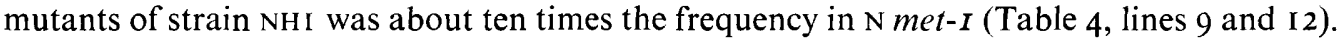

\section{Ultraviolet irradiation of transducing lysates}

Irradiation of the HFT lysates produced a simple exponential decline in the frequencies of ampicillin and/or kanamycin resistance transduction in strains $\mathrm{N}$ and $\mathrm{NHI}$ as a function of time of irradiation (Fig. I). This was taken to mean that transduction proceeded by lysogenization and not by double cross-over events (Luria et al., 1960; Coetzee, 1974) between phage markers and the chromosomes of strains $\mathrm{N}$ or NHI.

\section{Lysogenic status of $\mathrm{PM} 804$}

No phage which plated on PM5006 could be isolated from PM804 by u.v. or mitomycin C induction. Treated cultures did not undergo the clearing suggestive of phage or bacteriocin liberation. Also, spontaneous plaques were never observed on lawns of PM804 and there was thus no evidence for PM804 being cryptically or frankly lysogenic (Krizsanovich, 1973).

\section{Properties of transductants}

All transductants of PM804 by both HFT lysates were lysogenic, in that they spontaneously liberated phage which plated on PM5006 in low titre (Table 5, line I). Ultraviolet induction raised the titre to about $10^{7}$ p.f.u./ml. Phage liberation occurred even with the few transductants which arose from experiments at a phage m.i. of $10^{-2}$ in which secondary infection was prevented. This phage had no visible effect on PM804, and when grown on PM5006 it did not transduce antibiotic resistance to any of the strains used. Transductants of PM804 could not be tested for immunity to lysis by homologous phage because the HFT phages had no visible effect even on the wild-type strain (Table 2). Transductants of strains $\mathrm{N}$ and $\mathrm{NHI}$, produced at a m.o.i. less than $10^{-4}$, were non-lysogenic in that they did not liberate, either spontaneously or following u.v. induction, phage which plated on PM5006 (Table 5, lines 2 and 4 ). Strain $N$ transductants were also susceptible to 'spot' formation (Table 5 , line 2) by the original lysates as well as phage 5006M and adsorbed the phages to the same 


\section{Table 5. Retransduction of kanamycin-resistant transductants by phage 5006 MHFTak lysate}

Transductions were done as outlined in the legend to Table 4.

$\begin{array}{lccc}\begin{array}{c}\text { Recipient } \\ \text { transductant }\end{array} & \begin{array}{c}\text { Susceptibility to } \\ \text { 5006MHFTk lysate }\end{array} & \begin{array}{c}\text { Phage } \\ \text { liberation* }\end{array} & \text { M.o.i. } \\ \mathrm{PM} 804(\overline{\mathrm{K}})+ & \mathrm{NVE} & + & 2 \S \\ \mathrm{N}(\overline{\mathrm{K}}) & \mathrm{NPO} & - & \mathrm{I} \cdot 4 \\ \mathrm{~N}(\overline{\mathrm{K}}) & \mathrm{NVE} & + & \mathrm{I} \cdot 2 \S \\ \mathrm{NHI}(\overline{\mathrm{K}}) & + & - & \mathrm{I} \cdot 2 \\ \mathrm{NHI}(\overline{\mathrm{K}}) & \mathrm{NVE} & + & \mathrm{I} \cdot 4 \S\end{array}$

Transduction
frequency
A
3
$4 \times 10^{-5}$
1 I
$2 \times 10^{-4}$
32

32

NPO, Areas of partial clearing which disappeared on dilution of the lysate, no plaques observed; NVE, no visible effect; other symbols as in Table 2.

* Plating on PM5006.

$\dagger$ Transduction frequency/p.f.u. adsorbed, or number of transductants to ampicillin resistance.

¥ Superior line indicates that marker was transduced.

$\S$ Multiplicity input.

extent as strain N. Strain NHI transductants plated the phages (Table 2, line 25). At higher m.o.i., lysogenic transductants were obtained (Table 5, lines 3 and 5). These liberated phage and were converted (Coetzee, 1974) to non-adsorption of homologous phage (Table 2, line 24), just as single infection of NHI by phage $5006 \mathrm{M}$ converted it to non-adsorption of the phage or its HFT derivatives (Table 2, line 26).

At least $10 \%$ of clone-forming bacteria present in overnight broth cultures of all transductants had segregated the transduced markers. Segregants of phage 5006MHFTak transductants selected for sensitivity to ampicillin were invariably sensitive to kanamycin, and vice versa. The frequency of segregation was indicative of transduction by lysogenization in all instances, and transductants were thus heterogenote-like (Luria et al., 1960; Coetzee, 1974; see also Woods \& Thomson, 1975). This conclusion was supported by the effect u.v. irradiation of the transducing lysates had on transduction frequencies (Fig. I). Kanamycinresistant lysogenic transductants of PM804 could sometimes be retransduced to ampicillin resistance" by phage 5006MHFTak lysates (Table 5, line I) but the number of transductants was similar to the original transduction with PM804 as recipient (Table 4, lines I to 6). Kanamycin-resistant lysogenic and non-lysogenic transductants of strains N and NHI could be retransduced to ampicillin resistance by phage 5006MHFT $a k$ lysates (Table 5, lines 2 to 5 ). Retransduction frequencies could not be determined for transductions to the lysogens as phage adsorption could not be demonstrated but there were more ampicillin-resistant transductants with strain $\mathrm{NHI}(\overline{\mathrm{K}})$ than with $\mathrm{N}(\overline{\mathrm{K}})$ lysogens, in keeping with primary transductions to strains NHI and N, respectively. The phage adsorbed avidly to non-lysogenic kanamycin-resistant transductants and transduction of ampicillin resistance proceeded at frequencies (Table 5, lines 2 and 4) comparable to those at which strains $\mathrm{N}$ and NHI were transduced (Table 4, lines 8 and I2). At low m.o.i. of superinfecting transducing phage, double transductants of strain NHI were obtained which adsorbed and plated phage $5006 \mathrm{M}$.

\section{Properties of phage derived from transductants}

Ultraviolet-induced lysates of PM804 antibiotic-resistant transductants regularly produced many more PM804 transductants than phage 5006MHFT $k$ or 5006MHFTak lysates (Table 6, lines I and 2; Table 4, lines I to 6) but transduced PM5006 to antibiotic resistance at much lower frequencies than lysates of the latter two phages (Table 6, line 3). Phage in the PM804 
Table 6. Transducing ability of u.v.-induced lysates from $\mathrm{PM} 804, \mathrm{~N}$ and $\mathrm{NHI}$ transductants

Transductions were done as outlined in the legend to Table 4 .

\begin{tabular}{|c|c|c|c|c|}
\hline \multirow[b]{2}{*}{ Phage lysate } & \multirow[b]{2}{*}{ Recipient } & \multirow[b]{2}{*}{ M.o.i. } & \multicolumn{2}{|c|}{$\begin{array}{l}\text { Transduction frequency } \\
\text { p.f.u. adsorbed or } \\
\text { no. of transductants }\end{array}$} \\
\hline & & & $\mathrm{K}$ & $\mathrm{AK}$ \\
\hline 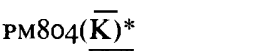 & PM804 & $3 \dagger$ & 25 & - \\
\hline PM804( $\overline{\mathrm{AK}})$ & PM804 & $3 \dagger$ & 17 & 24 \\
\hline $\mathrm{PM} 804(\overline{\mathrm{AK}})$ & PM5006 & $I \cdot 2$ & - & $10^{-5}$ \\
\hline 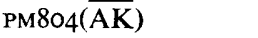 & & 3 & & \\
\hline$\stackrel{+}{\text { 5006М.PM5006 str-r }}$ & PM5006 & 2 & - & $3 \times 10^{-5}$ \\
\hline $\begin{array}{c}\mathrm{PM} 804(\overline{\mathrm{AK}}) \\
+\end{array}$ & PM5006 & 3 & - & $3 \times 10^{-5}$ \\
\hline 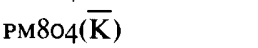 & & 15 & & \\
\hline PM804(AK) & 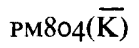 & $2 \dagger$ & - & 36 \\
\hline $\mathrm{N}(\overline{\mathrm{AK}})$ & $\mathbf{N}$ & 0.9 & - & $5 \times 10^{-5}$ \\
\hline $\mathrm{N}(\overline{\mathrm{AK}})$ & $\mathrm{NHI}$ & 0.8 & - & $1 \times 10^{-4}$ \\
\hline NHI $(K)$ & $\mathrm{N}$ & 0.3 & $10^{-5}$ & - \\
\hline $\mathrm{N}(\overline{\mathrm{AK}})$ & PM5006 & $I \cdot 2$ & - & $5 \times 10^{-2}$ \\
\hline
\end{tabular}

For symbols, see Table 2.

* Superior line indicates that markers were transduced.

$\dagger$ Multiplicity input.

transductant lysates could not be demonstrated to adsorb to PM804, while $>99 \%$ adsorption occurred to PM5006, N and NHI (Table 2, lines I6 to 19). Simultaneous infection with phage 5006M.PM5006 str- $r$ at a m.o.i. of 2 raised the PM804 lysate transduction frequency of antibiotic resistance to PM5006 about threefold (Table 6, line 4). Increase in the m.o.i. of the non-transducing phage had no further effect, and simultaneous infection (Table 6, line 5) with phage which bore an incompatible modification pattern did not further increase the transduction frequency to PM5006.

Failure to demonstrate phage adsorption to PM804 made it impossible to determine whether PM804 transductants were converted to homologous phage non-adsorption. However, PM804 kanamycin-resistant transductants could be retransduced to ampicillin resistance (Table 6, line 6), consistently and in greater numbers, by phage from PM804 transductants than with phage from PM5006 (Table 5 , line I); presumably one factor involved was the absence of lysogenic conversion in recipients. In a single-cycle growth experiment with PM5006 and phage present in PM804 transductant lysates, transmission was severely curtailed despite the fact that the lysate still transduced the strain at a frequency of $10^{-5} /$ p.f.u. adsorbed (Table 6 , line 3). This meant that PM804 modified the original transducing phage so that phage produced by it was susceptible to a restriction system present in PM5006. The fact that phage from PM804 transductants consistently produced more transductants in PM804 (Table 6, lines I, 2 and 6) than the original phage (Table 4, lines I to 6) implied that PM804 also possessed a restriction system which recognized unmodified phage that happened to adsorb.

Uitraviolet-induced phage lysates of strains $\mathrm{N}$ and NHI transductants transduced $\mathrm{N}$ or NHI at frequencies similar to those of the original HFT phage lysates (Table 6, lines 7 to 9 ; Table 4, lines 7, 8 and $\mathrm{I}_{2}$ ). Transduction to PM5006 also occurred at frequencies similar to 
Table 7. Transduction of segregants of transductants

\begin{tabular}{|c|c|c|c|c|}
\hline \multirow[b]{2}{*}{ Phage lysate } & \multirow[b]{2}{*}{ Recipient } & \multirow[b]{2}{*}{ M.o.i. } & \multicolumn{2}{|c|}{$\begin{array}{l}\text { Transducting frequency } \\
\text { p.f.u. adsorbed or } \\
\text { no. of transductants }\end{array}$} \\
\hline & & & $\mathbf{K}$ & AK \\
\hline $5006 \mathrm{MHFT} k$ & $\mathrm{PM} 804(\overline{\mathbf{K}}) \mathrm{K}^{\mathrm{s} *}$ & $3 \dagger$ & 3 & - \\
\hline $5006 \mathrm{MHFT} a k$ & PM804 $(\overline{\mathrm{AK}}) \mathrm{A}^{\mathrm{s}} \mathrm{K}^{\mathrm{s}}$ & $3 t$ & I & 4 \\
\hline $\mathrm{PM} 804(\overline{\mathrm{AK}})$ & 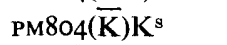 & $3 \dagger$ & 40 & 46 \\
\hline 5006MHFTak & $\mathrm{N}(\overline{\mathrm{AK}}) \mathrm{A}^{\mathrm{s}} \mathrm{K}^{\mathrm{s}}$ & 0.8 & - & $4 \times 10^{-5}$ \\
\hline 5006MHFT $a k$ & $\mathrm{NHI}(\overline{\mathbf{K}}) \mathrm{K}^{\mathrm{s}}$ & 0.7 & - & $10^{-4}$ \\
\hline $5006 \mathrm{MHFT} a k$ & $\mathrm{NHI}(\overline{\mathrm{K}} \overline{\mathrm{AK}}) \mathrm{A}^{\mathrm{s}}$ & 0.7 & - & $10^{-4}$ \\
\hline $\mathrm{N}(\overline{\mathrm{AK}})$ & $\mathrm{N}(\overline{\mathbf{K}}) \mathrm{K}^{\$}$ & 0.7 & - & $3 \times 10^{-5}$ \\
\hline
\end{tabular}

For symbols, see Table 2.

* Superior line indicates that marker was transduced; superior s signifies segregant of particular marker.

$\uparrow$ Multiplicity input.

those produced by these lysates (Table 6 , line I0). These results were anticipated as the strains did not modify phage from PMI 3 (Coetzee \& Smit, 1969) or PM5006 (Table 3, line I).

The segregation properties of kanamycin-resistant transductants (PM804( $(\bar{K})$ ) retransduced to ampicillin and kanamycin resistance $(\mathrm{PM} 804(\overline{\mathrm{K}} \overline{\mathrm{AK}})$ ) by u.v.-induced lysates of PM804 $(\overline{\mathrm{AK}})$ were investigated. While overnight broth cultures of these double transductants contained an average of $16.4 \%$ ampicillin-sensitive segregants, the cultures only produced an average of $2.3 \%$ kanamycin-sensitive clones. Thus the markers of each transducing phage segregated independently.

\section{Properties of segregants}

The lysogenic status of segregants was invariably similar to that of parent transductants. Segregants of $\mathrm{PM} 804_{4}$ transductants were transduced to antibiotic resistance by the phage lysates in similar numbers to those produced with PM804 as recipient (Table 7, lines I and 2; Table 4, lines I to 6). This was further evidence in favour of inability of the transducing phages to convert hosts to homologous phage non-adsorption. Just like transductants, segregants too were transduced by phage from PM804 transductants in greater numbers and more consistently (Table 7 , line 3 ; Table 6, line 6) than PM804 was transduced by lysates $5006 \mathrm{MHFT} k$ or $5006 \mathrm{MHFT} a k$ (Table 4 , lines I to 6 ). The fact that lysates of PM804( $\overline{\mathrm{AK}}$ ) produced similar numbers of transductants with recipients $\mathrm{PM} 804(\overline{\mathrm{K}})$ as with segregant PM804 $(\overline{\mathrm{K}}) \mathrm{K}_{\text {: }}^{\text {s }}$ (Table 6, line 6; Table 7 , line 3 ) could have meant that phage 5006MHFTak did not integrate in the kanamycin resistance region of the resident prophage. Non-lysogenic segregants of strains $\mathrm{N}$ and $\mathrm{NHI}$ transductants were transduced to antibiotic resistance at frequencies similar to those at which strains $\mathrm{N}$ and $\mathrm{NHI}$ were transduced (Table 7 , lines 4 and 5 ; Table 4 , lines 8 and $\mathrm{I} 2$ ). Transduction of ampicillin resistance with phage 5006MHFT $a k$ to ampicillin-sensitive kanamycin-resistant single segregants of double non-lysogenic transductants of strain NHI also occurred at frequencies similar to those of ampicillin plus kanamycin resistance transduction to NHI by phage 5006MHFTak lysates (Table 7, line 6; Table 4, line 12). It is noteworthy that the homology offered by resident prophage 5006 MHFT $k$ did not affect transduction frequencies. Also, u.v.-induced lysates of strain $\mathrm{N}$ transductants transduced strain $\mathrm{N}$ non-lysogenic segregants at the same frequencies as phage 5006 MHFTak (Table 7 , lines 4 and 7 ). This was confirmation that strain $\mathrm{N}$ failed to modify phage from PM5006 (Table 3 , line I). 
Heat treatment of strains

Due to poor adsorption, the effect of heat treatment of PM804 on phage transmission could not be tested. Treatment of strain $\mathrm{N}$ for periods of 5 and 20 min at various temperatures between 40 and $65^{\circ} \mathrm{C}$ immediately before use in transmission experiments with a 5006MHFTak phage lysate did not increase transmission frequencies above that recorded in Table 3 , line $\mathrm{I}$. Heat treatment of PM804 or strain $\mathrm{N}$ before use in transduction experiments with HFT lysates did not make results with PM804 more predictable or increase the number of transductants, nor were transduction frequencies of markers to strain $\mathrm{N}$ increased.

\section{DISCUSSION}

The selection provided by the high frequency antibiotic resistance transducing phages derived from $P$. mirabilis strain PM5006 has revealed additional hosts, $P$. vulgaris strain PVI 27 (Coetzee, I975b) and $P$. mirabilis PM804, on which the phage lysates do not have macroscopic effects. These hosts have permitted transduction across a species barrier and the Dienes incompatibility line (Dienes, 1946, 1947; see Coetzee, 1972).

Use of strain NHI provided transductants which for the first time (Coetzee, 1974, 1975a, $b$ ) proved to be non-lysogenic, and which were heterogenote-like and segregated markers at high frequency. In these properties they resembled the single particle transductants produced by specialized variants of phage P22 (Hoppe \& Roth, 1974) but differed from the phagesensitive transductants produced by single infection with HFT lysates of phage lamda (Campbell, 1957) which were stable rather than heterogenotic. Here then was evidence for a defect in a maturation function of the HFT phages in that they could not register as plaqueforming particles (see Coetzee, $1975 b$ ). Transductants of strain NHI confirmed a suggestion (Coetzee, 1975b) that both the HFT phages were defective in conversion of hosts to nonadsorption of homologous phages. There is no evidence that NHI carries a related prophage (see Coetzee \& Smit, I969) and single infection by the parent phage 5006M was clearly capable of converting NHI to phage non-adsorption (Table 2, line 26). Defectiveness of the conversion gene(s) was supported by recovery of serial double transductants of NH I which still adsorbed and plated homologous phage: provided the prophages were situated in tandem on the chromosome, this mode of integration clearly did not assist in reconstituting a functional gene.

The observation that HFT lysates transduced lysogenic transductants in the absence of demonstrable adsorption implied that measurement of phage adsorption was not as sensitive as transductant selection and that lysogenic conversion to phage non-adsorption did not constitute an absolute barrier to homologous phage. Despite the fact that phage 5006MHFTak carries an additional marker, no other difference was detected between it and phage $5006 \mathrm{MHFT} k$.

The failure to detect abortive transductants of antibiotic resistance with all three recipients corresponds to results obtained with transductions of these markers to $P$. vulgaris strain PVI 27 (Coetzee, I975b). If there was sufficient microhomology in a $P$. vulgaris strain for the phages to integrate, it could be assumed that this also held true for the $P$. mirabilis hosts studied here.

The reason why all PM804 transductants were lysogenic for phage plating on PM5006 is obscure. The absence of demonstrable phage adsorption to PM804, coupled with the use of low phage input, ensured that transductants arose from single phage infection. As there was no evidence for strain PM804 being cryptically lysogenic, the explanation given previously 
(Coetzee, 1974) for all PM5006 transductants by phage 5006MHFT $k$ being frankly lysogenic, does not apply.

The fact that lysates of PM804 transductants yielded transducing phage which adsorbed well to PM5006 but could not be shown (by adsorption tests) to adsorb to PM804, ruled out host range mutants of the phages as the cause of the original PM804 transductants. The phenotype of PM804 differed from that of $P$. vulgaris strain PVI27 which did not modify the $P$. mirabilis transducing phages (Coetzee, I $975 b$ ). The transducing lysates of 5006MHFT $k$ and 5006MHFTak thus not only adsorbed poorly to PM804 but had to contend with the restriction system of the latter organism. While the first obstacle remained, transducing phage derived from PM804 transductants possessed a compatible modification pattern. Taken in conjunction with the fact that the phage in single infection did not convert PM804 to homologous phage non-adsorption, this could explain why phage from PM804 transduced PM804 more readily than the original phages from PM5006. Due to the high potential transduction frequency, these lysates also retransduced appropriate lysogenic transductants despite possible lysogenic conversion to phage non-adsorption. Thus strain PM804, like PVI27 (Coetzee, I975b) was not an ideal host for determining the properties of the HFT phage lysates.

In contrast to PM804 were strain $\mathrm{N}$ and its restrictionless mutant $\mathrm{NHI}$; both appear to be modification-deficient, and lysates of transductants of these strains transduced PM5006 at high frequency (see Table 6, line Io) while the parent strains were transduced at frequencies similar to those of the phage 5006MHFT $k$ and 5006MHFTak lysates respectively (Table 4, lines 8 and 12 ; Table 6, lines 7,8 and 9).

Despite the low transmission of the HFT phages in strain $\mathrm{N}$, the transduction frequencies to strain $\mathrm{N}$ were never $<10^{-1}$ of corresponding frequencies to strain $\mathrm{NHI}$ in which transmission was $100 \%$ (Table 3 , line 2). This phenomenon was noted with general transduction to these strains (Coetzee \& Smit, 1969) and could mean that the particular markers transduced were protected from restriction enzymes, or possibly that transduced genetic material lacked sites susceptible to restriction enzymes of strain $\mathrm{N}$.

The cause of the increase in transduction frequencies which simultaneous infection with non-transducing phage effected was most likely to have been the result of a helper effect rather than a multiplicity effect (Lederberg, I965; Coetzee, I974; see also Uetake, Toyama $\&$ Hagiwara, 1964). This was supported by the fact that non-transducing phage which bore an incompatible modification pattern did not have a greater effect than compatible phage and that maximal effects were attained at a m.o.i. of about 3. The fact that transduction frequencies to strain NHI (which is restrictionless) were increased to the same extent and at similar m.o.i. by this phage, also favoured a helper effect. The helper effect was small in comparison with that extended in transductions by the HFT phages to the homologous host PM5006 (Coetzee, 1974, 1975a). For strain $\mathrm{N}$ this result could (in the absence of a multiplicity effect) be due to the fact that phage $5006 \mathrm{M}$ was also restricted, but this would not apply to strain NHI. However, the latter strain was derived from strain N by nitrosoguanidine treatment and changes other than merely the selected mutation could be involved (see Coetzee \& Smit, I969). For instance, another discrepancy noted with strain NHI was that phage 34. NHI had an e.o.p. of $5 \times 10^{-4}$ on strain $\mathrm{N}$ (Coetzee \& Smit, 1969) while u.v.induced lysates of lysogenic strain NHI transductants did not form plaques on the former strain (Table 2, line 23).

Another peculiarity of the systems investigated was that postulated restriction endonucleases could not be demonstrated to be heat susceptible.

The author is in receipt of grants from the South African Medical Research Council. 


\section{REFERENCES}

Adams, M. H. (1956). Methods of study of bacterial viruses. Methods in Medical Research 2, I-73.

Adelberg, E. A., Mandel, M. \& Chen, G. C. C. (I965). Optimal conditions for mutagenesis by $N$-methyl$N^{\prime}$-nitro- $N$-nitrosoguanidine in Escherichia coli $\mathrm{KI}$ 2. Biochemical and Biophysical Research Communications $\mathbf{1 8}, 788-795$.

Altenbern, R. A. \& Stull, H. B. (1965). Inducible lytic systems in the genus Bacillus. Journal of General Microbiology 39, 53-62.

Campbell, A. (1957). Transduction and segregation in Escherichia coli K12. Virology 4, 366-384.

Coetzee, J. N. (196I). Lysogenic conversion in the genus Proteus. Nature, London 189, 946-947.

Coetzee, J. N. (1972). Genetics of the Proteus group. Annual Review of Microbiology 26, $23-54$.

Coetzee, J. N. (1974). High frequency transduction of kanamycin resistance in Proteus mirabilis. Journal of General Microbiology 84, 285-296.

Coetzee, J. N. (I975a). High frequency transduction of ampicillin and kanamycin resistance in Proteus mirabilis. Journal of General Microbiology 87, 173-176.

Coetzee, J. N. (I975b). Transduction of a Proteus vulgaris strain by a Proteus mirabilis bacteriophage. Journal of General Microbiology 89, 299-309.

Coetzee, J. N., Datta, N. \& Hedges, R. W. (1972). R factors from Proteus rettgeri. Journal of General Microbiology 72, 543-552.

Coetzee, J. N., Datta, N., Hedges, R. W. \& Appelbaum, P. C. (1973). Transduction of R factors in Proteus mirabilis and $P$. rettgeri. Journal of General Microbiology 76, 355-368.

Coetzee, H. L., de KLeRK, H. C. \& Coetzee, J. N. (1968). Bacteriophage-tail-like particles associated with intra-species killing of Proteus vulgaris. Journal of General Virology 2, 29-36.

Coetzee, J. N. \& SACks, T. G. (1960). Transduction of streptomycin resistance in Proteus mirabilis. Journal of General Microbiology 23, 445-455.

Coetzee, J. N. \& Smit, J. A. (1969). Restriction of a transducing bacteriophage in a strain of Proteus mirabilis. Journal of General Virology 4, 593-607.

Coetzee, J. N. \& Smit, J. A. (1970). Properties of Proteus mirabilis phage i 3vir. Journal of General Virology 9, 247-249.

CORNElis, G. \& Colson, C. (1975). Restriction of DNA in Yersinia enterocolitica detected by recipient ability for a derepressed R factor from Escherichia coli. Journal of General Microbiology 87, 285-291.

Dienes, L. (1946). Reproductive processes in Proteus cultures. Proceedings of the Society for Experimental Biology and Medicine 63, 265-270.

Dienes, L. (1947). Further observations on the reproduction of bacilli from large bodies in Proteus cultures. Proceedings of the Society for Experimental Biology and Medicine 66, 97-98.

Goldberg, R. B., Bender, R. A. \& Streicher, S. L. (1974). Direct selection for Pi-sensitive mutants of enteric bacteria. Journal of Bacteriology I18, 810-814.

Grabow, W. I. K. \& Smit, J. A. (1967). Methionine synthesis in Proteus mirabilis. Journal of General Microbiology 46, 47-57.

Hoppe, I. \& Roth, J. (I974). Specialized transducing phages derived from Salmonella phage P22. Genetics 76, 633-654.

Kondo, E. \& Mitsuhashi, S. (I 966$)$. Drug resistance of enteric bacteria. VI. Introduction of bacteriphage PICM into Salmonella typhi and formation of PrdCM and F-CM elements. Journal of Bacteriology 9r, $1787-1794$.

Krizsanovich, K. (1973). Cryptic lysogeny in Proteus mirabilis, Journal of General Virology 19, 31 I-320.

Lederberg, S. (I965). Host-controlled restriction and modification of deoxyribonucleic acid in Escherichia coli. Virology 27, 378-387.

Luria, S. E., Adams, J. N. \& Ting, R. C. (1960). Transduction of lactose-utilizing ability among strains of $E$. coli and $S$. dysenteriae and the properties of the transducing phage particles. Virology 12, 348-390.

Morse, M. L., Lederberg, E. M. \& LederberG, J. (1956). Transductional heterogenotes in Escherichia coli. Genetics 4r, 758-779.

Uetake, H., Toyama, S. \& Hagiwara, S. (I964). On the mechanism of host-induced modification. Multiplicity activation and thermolabile factor responsible for phage growth restriction. Virology 22, $202-2 \mathrm{I} 3$.

Woods, D. R. \& Thomson, J. A. (1975). Unstable generalized transduction in Achromobacter. Journal of General Microbiology 88, 86-92.

Yamamoto, K. R. \& Alberts, B. M. (1970). Rapid bacteriphage sedimentation in the presence of polyethylene glycol and its application to large-scale virus purification. Virology 40, 734-744. 